\title{
Convergent animal and human evidence suggests the activin/inhibin pathway to be involved in antidepressant response
}

\author{
K Ganea ${ }^{1}$, A Menke ${ }^{1}$, MV Schmidt ${ }^{1}$, S Lucae ${ }^{1}$, G Rammes ${ }^{1,2}$, C Liebl $^{1}$, D Harbich ${ }^{1}$, V Sterlemann ${ }^{1}$, C Storch ${ }^{1}$, M Uhr ${ }^{1}$, F Holsboer ${ }^{1}$, \\ EB Binder ${ }^{1}$, I Sillaber ${ }^{3}$ and MB Müller ${ }^{1}$
}

Despite the overt need for improved treatment modalities in depression, efforts to develop conceptually novel antidepressants have been relatively unsuccessful so far. Here we present a translational approach combining results from hypothesis-free animal experiments with data from a genetic association study in depression. Comparing genes regulated by chronic paroxetine treatment in the mouse hippocampus with genes showing nominally significant association with antidepressant treatment response in two pharmacogenetic studies, the activin pathway was the only one to show this dual pattern of association and therefore selected as a candidate. We examined the regulation of activin A and activin receptor type IA mRNA following antidepressant treatment. We investigated the effects of stereotaxic infusion of activin into the hippocampus and the amygdala in a behavioural model of depression. To analyse whether variants in genes in the activin signalling pathway predict antidepressant treatment response, we performed a human genetic association study. Significant changes in the expression of genes in the activin signalling pathway were observed following 1 and 4 weeks of treatment. Injection of activin $A$ into the hippocampus exerts acute antidepressant-like effects. Polymorphisms in the betaglycan gene, a co-receptor mediating functional antagonism of activin signalling, significantly predict treatment outcome in our system-wide pharmacogenetics study in depression. We provide convergent evidence from mouse and human data that genes in the activin signalling pathway are promising novel candidates involved in the neurobiogical mechanisms underlying antidepressant mechanisms of action. Further, our data suggest this pathway to be a target for more rapid-acting antidepressants in the future.

Translational Psychiatry (2012) 2, e177; doi:10.1038/tp.2012.104; published online 23 October 2012

\section{Introduction}

Affective disorders are a leading cause of morbidity and mortality worldwide. ${ }^{1,2}$ Although currently available antidepressant drugs are safe and effective, the vast majority of patients do not respond until 3-5 weeks after the initiation of treatment, with only $<50 \%$ of them achieving remission with the first compound prescribed. ${ }^{3}$

However, despite the overt need for improved treatment modalities, including compounds with a more rapid onset of action, ${ }^{4}$ efforts to develop conceptually novel antidepressants have been relatively unsuccessful so far. ${ }^{5}$ As it has been hypothesized that the individual genetic make-up contributes to the striking heterogeneity in antidepressant response, the identification of genetics factors that might assist in the prediction of an individual's drug response has attracted increasing attention during the past years. For candidatedriven approaches, distinct single-nucleotide polymorphisms (SNPs) have been shown to predict specific aspects of antidepressant treatment response in affective disorder. ${ }^{6-9}$ However, despite tremendous efforts in identifying novel, yet unexplored predictive genes in large genome-wide association studies, the results are fairly modest. None of the SNPS identified in those studies finally achieved genome-wide significance or was consistently replicated across studies, suggesting that alternative pharmacogenetic strategies need to be established. ${ }^{10,11}$

In this study, we use a conceptually different approach by integrating and combining data from hypothesis-free animal experiments applying antidepressant treatment conditions comparable to the clinical situation (that is, chronic oral paroxetine treatment) ${ }^{12}$ with data from an association study for antidepressant treatment response in depressed patients. ${ }^{13}$ We provide convergent evidence for an involvement of members of the activin signalling pathway in antidepressant treatment response. This latter pathway was selected because it was the only one to show significant regulation by chronic paroxetine treatment in the mouse hippocampus and to show nominally significant association with antidepressant treatment response in both the Munich Antidepressant Response Signature (MARS) projects as well as STAR*D. ${ }^{13}$

We here demonstrate that activin $A$ has antidepressant-like properties in vivo. The time course of paroxetine-induced regulation of activin $\beta A$ together with its acute antidepressant-

\footnotetext{
${ }^{1}$ Max Planck Institute of Psychiatry, Munich, Germany; ${ }^{2}$ Department of Anesthesiology, Technische Universtität, Munich, Germany and ${ }^{3}$ Phenoquest AG, Martinsried/ Munich, Munich, Germany

Correspondence: Dr MB Müller, Molecular Stress Physiology, Max Planck Institute of Psychiatry, Kraepelinstr. 2-10, 80804 Munich, Germany.

E-mail: muellerm@mpipsykl.mpg.de

Keywords: activin; antidepressants; depression; gene expression profiling; SNP

Received 7 February 2012; revised 26 July 2012; accepted 4 September 2012
} 
like properties in a behavioural model of depression point to a potentially more rapid onset of action. Translating our preclinical findings to the human situation, we are finally able to provide evidence that the activin system has a role in response to antidepressant drug treatment in depressed patients: in a genetic association study, we identify specific polymorphisms in the betaglycan gene, a co-receptor binding inhibin and mediating functional antagonism of activin signalling, ${ }^{14}$ to significantly predict antidepressant drug treatment outcome in depression.

\section{Materials and methods}

Gene selection criteria. To identify strong candidates in this translational approach, we compared genes that were shown to be significantly regulated by chronic paroxetine treatment in the mouse hippocampus (see Sillaber et al. ${ }^{12}$ and Supplementary Table 1), with the 43 genes showing nominally significant association with antidepressant treatment response in both the MARS projects as well as STAR*D (see Ising et al. ${ }^{13}$ and Supplementary Table 1) In fact, the activin pathway was the only pathway that showed this pattern of dual association (mouse/human) and was therefore chosen for further analysis.

Animals and housing conditions. Experiments were carried out with male DBA/2OlaHsd mice (age: 10-16 weeks; Harlan Winkelmann, Eystrup, Germany) that were singly housed in standard cages under a 12L:12D cycle and constant temperature $\left(23 \pm 2{ }^{\circ} \mathrm{C}\right)$ conditions. Food and water were provided ad libitum. The experiments were approved by the committee for the Care and Use of Laboratory Animals of the Government of Upper Bavaria, Germany.

Antidepressant treatment. Animals were randomly distributed to the vehicle or paroxetine treatment group. Paroxetine (Seroxat; GlaxoSmithKline, Brentford, UK) was suspended in water to a final concentration of $1 \mathrm{mg} \mathrm{ml}^{-1}$. The drug $\left(10 \mathrm{mg} \mathrm{kg}^{-1}\right.$ ) or vehicle (water) were given orally by gavage twice per day over a period of 28 days (chronic experiment), 7 days (subchronic schedule) or only once (acute treatment). In all cases, on the last day (or on the day of single acute treatment, respectively), the animals were treated in the morning at 0800 hours and were killed $4 \mathrm{~h}$ later. Brains were removed, frozen in isopentane at $-40^{\circ} \mathrm{C}$ and stored at $-80^{\circ} \mathrm{C}$ for in situ hybridization studies.

In situ hybridization and semiquantitative analysis of mRNA expression levels. Frozen brains were sectioned in a cryostat microtome ( $16 \mu \mathrm{m}$, coronal sections) and sections were kept at $-80^{\circ} \mathrm{C}$ until use. In situ hybridization using a ${ }^{35} \mathrm{~S}$-UTP-labelled ribonucleotide probes were performed as described previously. ${ }^{15}$ The activin $\beta$ A cDNA (508bp) was generated by PCR amplification from mouse hippocampus and subsequently cloned into a pCRII-TOPO vector (Invitrogen, Carlsbad, CA, USA). The primers used were as follows: activin $\beta A$ upstream (5'-TGGATGGAGATGGGAAGAAG-3') and activin $\beta A$ downstream (5'-TCCATTTTCTCTGGGA CCTG-3'). The cDNA template for activin $\beta A$, including T7 and SP6 promotors for sense and antisense riboprobe in vitro transcription, was generated by PCR amplification from the vector using a T7 primer (5'-GAATTGTAATACGAC TCACTATAGGGCGAATTG-3') and an SP6 primer (5'-CC AAGCTATTTAGGTGACACTATAGAATACT-3'). The activin receptor IA in situ probe was previously described in detail.

The slides were apposed to Kodak Biomax MR films (Eastman Kodak, Rochester, NY, USA) and developed. Autoradiographs were digitized, and relative expression was determined by computer-assisted optical densitometry (Scion Image; Scion Corporation, Frederick, MD, USA) as described previously. $^{16}$

Behavioural effects of stereotactic infusion of activin A into the dentate gyrus and amygdala. Mice were anesthetized with pentobarbital sodium diluted in $0.9 \% \mathrm{NaCl}$ (80 $\mathrm{mg} \mathrm{kg}^{-1}$ bodyweight, intraperitoneally; Narcoren; Rhone Merieux, Hallbergmoos, Germany) and placed in a stereotactic apparatus (Stoelting, Wood Dale, IL, USA). Stainlesssteel guide cannulas (Hamilton, 23 gauge, Bonaduz, Switzerland) were bilaterally implanted (coordinates for the amygdala relative to bregma: $-1.0 \mathrm{~mm}$ anterior-posterior; $\pm 3.1 \mathrm{~mm}$ lateral and $-1.8 \mathrm{~mm}$ dorsoventral; for the dentate gyrus: $1.4 \mathrm{~mm}$ anterior-posterior, $\pm 1.0 \mathrm{~mm}$ lateral and $-1.1 \mathrm{~mm}$ dorsoventral, according to the Paxinos and Watson brain atlas). After surgery, animals were allowed to recover for 14 days. Mice were bilaterally infused with either recombinant human/mouse/rat activin $\mathrm{A}(1 \mu \mathrm{g}$ per side; carrier free; R\&D Systems, Minneapolis, MN, USA) in $0.1 \%$ bovine serum albumin in phosphate-buffered saline or vehicle. A total volume of $1 \mu \mathrm{l}$ was infused (Hamilton injection syringes, 31 gauge). Correct cannula placement was determined by post-mortem histological verification.

Modified hole board. To get a comprehensive overview on activin A-induced behavioural changes and to exlcude changes in basal locomotor activity, we performed the modified hole board test (for details see Ohl et al. ${ }^{17}$ ) The modified hole board enables the stress-free investigation of different behavioural dimensions in rodents in only one test. Mice received a single infusion of vehicle or activin $A$ and were tested in the modified hole board $20 \mathrm{~h}$ after the treatment. Board visits, rearing, grooming behaviour and stretched attends were manually scored by an experienced rater. Total covered distance (locomotor activity) was analysed automatically (Any-Maze Video Tracking System, Wood Dale, IL, USA).

Porsolt forced swim test. Mice received a bilateral infusion of either vehicle or activin A 15 min before the first trial of the forced swim test (FST) (5-min test period). ${ }^{18}$ The parameters swimming, struggling and floating were scored by a trained observer blind to the treatment. To investigate the effects of a first FST exposure under vehicle or activin A treatment on the stresscoping strategy, all animals were retested $24 \mathrm{~h}$ after the infusion.

Human genetic association study. To investigate whether variants (SNPS) in genes involved in the activin signalling pathway are associated with antidepressant treatment response, we performed a human genetic association study. 
Subjects. In all, 575 unipolar depressed patients (262 men and 313 women) aged 18 to 75 years who were admitted to the hospital of the Max Planck Institute of Psychiatry, Munich, Germany, were enroled within the MARS project (http://www.mars-depression.de). ${ }^{19,13,20}$ This is a naturalistic pharmacogenetic study and all patients are treated with antidepressants according to doctor's choice. Plasma antidepressant drug concentrations were monitored to ensure clinically efficient drug levels. Patients were diagnosed by psychiatrists according to the Diagnostic and Statistical Manual of Mental Disorders IV criteria and patients were included in the study within the first 3-5 days after in-patient admission. Patients with depressive disorder due to a general medical or neurological condition were excluded, as were patients with a lifetime diagnosis of drug abuse and depressive symptoms secondary to alcohol or substance abuse or dependency. We recorded ethnicity using a selfreport sheet for nationality, first language and ethnicity of the patient and of all four grandparents. The study was approved by the Ethics Committee of the Ludwig Maximilians University in Munich, Germany, and written informed consent was obtained from all subjects.

Severity of depressive symptoms was assessed at admission by trained raters using the 21 -item Hamilton Depression Rating Scale $\left(H A M-D^{21}\right)$. Patients fulfilling the criteria for at least a moderate depressive episode $(H A M-D \geqslant 14)$ were eligible. Ratings were performed within 5 days of admission and then weekly until discharge. Response to antidepressive treatment was assessed 5 weeks after admission and defined as a reduction $>50 \%$ in HAM-D scores from the score obtained at admission.

DNA preparation, SNP selection and genotyping. At the time of enrolment in the study, $40 \mathrm{ml}$ of ethylenediaminetetraacetic acid blood were drawn from each patient. DNA was extracted from fresh blood using the Puregene whole blood DNA extraction kit (Gentra Systems, Minneapolis, MN, USA).

SNPs were derived from a whole-genome association approach of our MARS pharmacogenetic study. ${ }^{13}$ Genotyping was performed on Illumina Human 610 quad bead chips (Illumina, San Diego, CA, USA) according to the manufacturer's standard protocols. This chip assays more than 620901 rationally selected tagging SNPs and copy number variation markers per sample. The average call rate exceeded $99 \%$, with samples below $98 \%$ being either retyped or excluded from the study. The reproducibility for samples genotyped twice was $99.99 \%$ or better. In all, 166 SNPs located in the genomic regions encoding genes involved in the activin signalling pathway, including markers up to $10 \mathrm{~kb} 5^{\prime}$ and $3^{\prime}$ of the transcription start and end, were selected in the following genes: follistatin ( $F S T$; $1 \mathrm{SNP}$ ), inhibin/activin beta $\mathrm{A}$ (INHBA; $1 \mathrm{SNP}$ ), inhibin alpha (INHA; $1 \mathrm{SNP})$, activin rezeptor 1A (ACVR1A; 20 SNPs), activin rezeptor 1B (ACVR1B; 4 SNPs), activin rezeptor $2 \mathrm{~A}$ ( $A C V R 2 A ; 8 \mathrm{SNPs})$, activin rezeptor 2B (ACVR2B; 4 SNPs), betaglycan (TGFBR3; 73 SNPs), Smad2 (SMAD2; 10 SNPs) and Smad3 (SMAD3; 44 SNPs).

\section{Statistical analysis}

Animal experiments. The commercially available program SPSS 12 was used for statistical analysis of all animal experimental data. Data from the modified hole board test (activin vs vehicle group) were analysed by a one-way analysis of variance (ANOVA). If a significant overall effect was found, ANOVA was followed by a post-hoc NewmanKeuls test. Comparisons of two groups were made by unpaired $t$-test. In all cases, the level of significance was set at $P<0.05$. Data are presented as mean \pm s.e.m.

Human genetic association study. Exact tests on HardyWeinberg equilibrium were performed for all SNPs. ${ }^{22}$ SNPs with a minor allele frequency below $10 \%$, with a call rate of $<98 \%$, or displaying Hardy-Weinberg equilibrium deviation at an error level below $10^{-5}$ were excluded from the analysis. The final analysis was performed with 127 SNPs.

Basic allelic tests were conducted using PLINK (http:// pngu.mgh.harvard.edu/purcell/plink). ${ }^{23}$ We used permutation procedures to generate significance levels empirically and adjusted all association for multiple testing using Bonferroni correction for 127 independent tests.

\section{Results}

The activin system is a target of chronic paroxetine treatment. Chronic antidepressant treatment with paroxetine significantly increased activin $\beta A$ mRNA expression in the CA1 $(P<0.05)$ and the dentate gyrus $(P<0.05$; Figure 1$)$. Paroxetine treatment had no effect on activin $\beta A$ mRNA level in the CA3 area of the hippocampus, the cortex and the thalamus (data not shown).

Activin is composed of homodimers or heterodimers of the $\beta$-subunits (for example, activin $A$ is a homodimer consisting of two activin $\beta A$ subunits), whereas inhibin is a dimeric protein composed of an $\alpha$ - and $\beta$-subunit. To exclude the possibility that the upregulation of activin $\beta A$ might lead to increased formation of inhibin $A$, which is a heterodimer consisting of an inhibin $\alpha$ and an activin $\beta A$ subunit, in situ hybridization for the inhibin $\alpha$ subunit was performed. Chronic paroxetine treatment resulted in a significant decrease of inhibin $\alpha$ mRNA levels in the dentate gyrus $(P<0.05)$, whereas no effects of paroxetine on inhibin $\alpha$ mRNA levels could be observed in the CA1 and the CA3 region of the hippocampus or the cortex (Figure 1). These results indicate that activin $A$, but not inhibin $A$, is a target of paroxetineinduced upregulation.

For activin receptor IA mRNA, a significant upregulation following chronic paroxetine treatment was observed in the hippocampal dentate gyrus as well as the CA3 region of the pyramidal cell layer $(P<0.05$; Figure 1$)$.

Subchronic, but not acute treatment with paroxetine increases activin $\beta A$ mRNA levels in the hippocampal dentate gyrus. Acute paroxetine treatment had no significant effect on activin $\beta A$ mRNA expression levels in any of the regions investigated (Figure 2). In contrast, subchronic treatment with paroxetine already resulted in an increase of activin $\beta A$ mRNA levels in the CA1 and the dentate gyrus of the hippocampus $(P<0.05)$. In the hippocampal CA3 region, a downregulation of activin $\beta A$ mRNA was observed $(P<0.05)$ (Figure 2). 

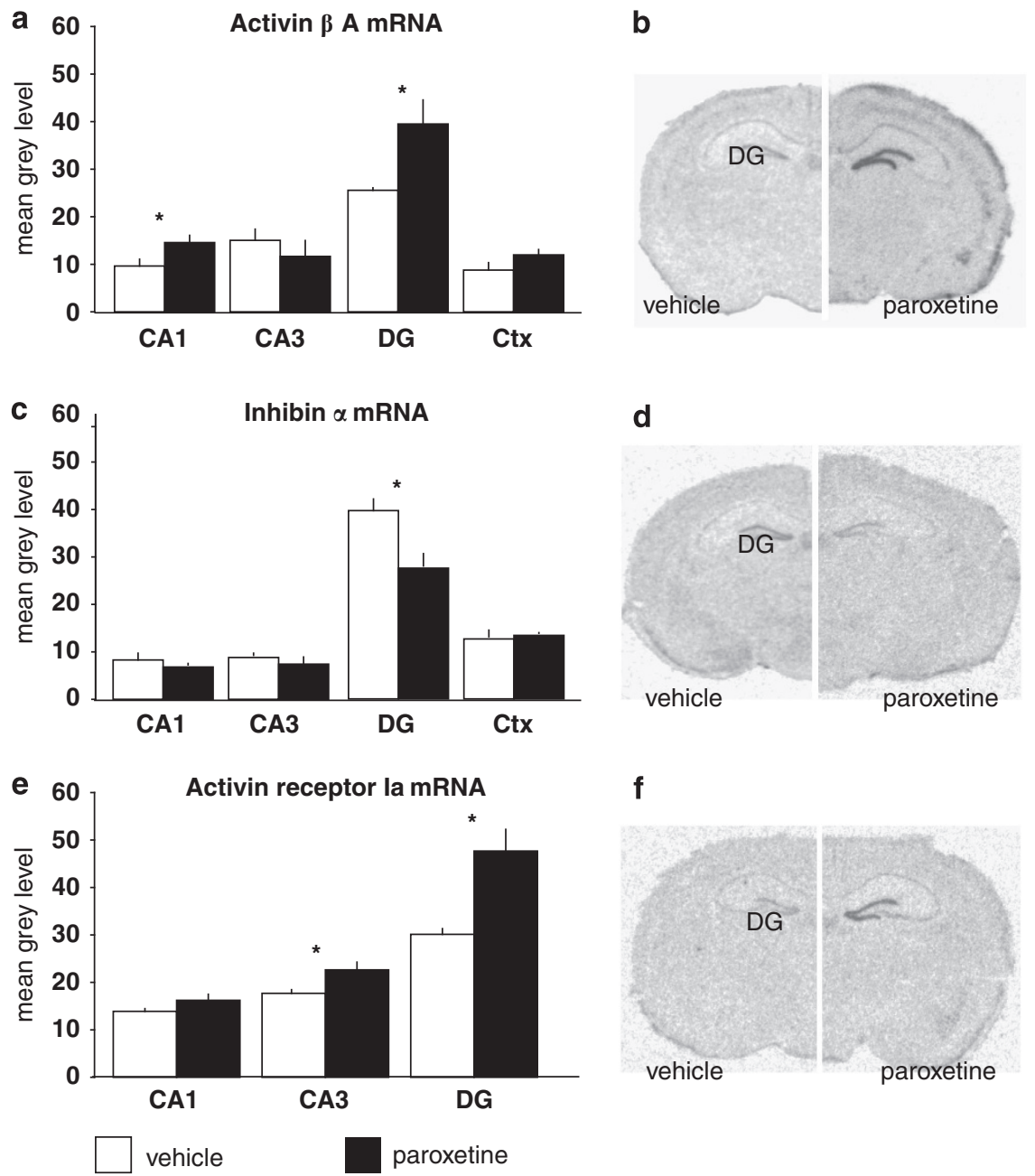

Figure 1 The activin system is a target of chronic treatment with the antidepressant paroxetine. (a) Chronic paroxetine treatment significantly increases the expression of activin $\beta A$ mRNA in the CA1 region of the hippocampal pyramidal cell layer (CA1) and the dentate gyrus (DG). (b) Representative autoradiographs illustrating the paroxetineinduced increase in hippocampal activin $\beta A$ mRNA expression compared with brains of vehicle-treated mice. (c and $\mathbf{d}$ ) Inhibin $\alpha$ mRNA, in contrast, was downregulated in the $D G$ following chronic paroxetine treatment. (a) Corresponding to the increase in activin $\beta A$ mRNA expression, chronic paroxetine induced the mRNA levels of activin receptor IA in the DG and the hippocampal CA3 region. (f) Representative autoradiographs illustrating the paroxetine-induced increase in activin receptor IA mRNA expression compared with brains of vehicle-treated mice. CA1, CA1 region of the hippocampal pyramidal cell layer; CA3, CA3 region of the hippocampal pyramidal cell layer; Ctx, cortex. Vehicle group: $n=8$; paroxetine group: $n=9$. ${ }^{*} P<0.05$.

\begin{abstract}
Stereotactic injection of activin A does not alter basal locomotor behaviour in the modified holeboard test. Injection of activin A bilaterally into either the amygdala or the dentate gyrus did not significantly alter any of the behavioural parameters scored in the modified holeboard test; in particular, there was no difference in general locomotor activity (total distance travelled) during $5 \mathrm{~min}$ of holeboard exposure (amygdala: vehicle, 17.12 \pm 3.3 ; activin A, $22.62 \pm 3.0$; dentate gyrus: vehicle, $21.4 \pm 7.4$; activin A, $24.5 \pm 6.1)$.
\end{abstract}

Stereotactic injection of activin A into the hippocampal dentate gyrus exerts region-specific antidepressant-like effects in the Porsolt FST. We observed a pronounced antidepressant-like effect of activin A injection into the dentate gyrus in the FST: animals treated with activin A showed an increase in struggling $(P<0.05)$ and a significant decrease in floating behaviour $(P<0.01)$ with no change in swimming (Figure 3a) $15 \mathrm{~min}$ after treatment. The significant difference in struggling behaviour between the treatment groups could still be observed when animals were retested $24 \mathrm{~h}$ after activin $\mathrm{A}$ injection $(P<0.05$; Figure $3 \mathrm{a})$.

Acute injection of activin $A$ into the amygdala did not significantly alter any of the behavioural parameters measured in the FST (Figure $3 b$ ). At the retest $24 \mathrm{~h}$ after the activin A injection, we observed a trend $(P=0.056)$ towards an increase in swimming behaviour in animals that had been injected with activin A into the amygdala compared with vehicle-injected animals with no effect on struggling or floating (Figure 3b).

Genetic variants in betaglycan, a member of the human activin system, predict antidepressant treatment response in depression. From 575 enroled depressed 

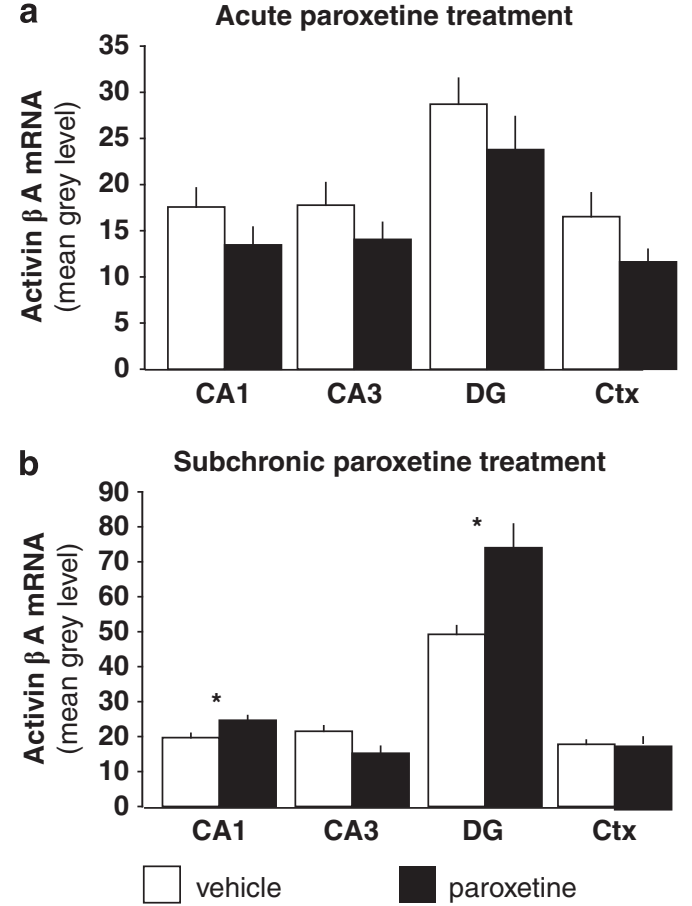

Figure 2 Regulation of hippocampal activin $\beta A$ mRNA expression is dependent on the duration of paroxetine treatment. (a) Acute treatment with paroxetine did not alter the expression level of activin $\beta A$ mRNA in any of the neuroantomical regions examined $(\mathrm{CA} 1=\mathrm{CA} 1$ region of the hippocampal pyramidal cell layer; $\mathrm{CA} 3=\mathrm{CA} 3$ region of the hippocampal pyramidal cell layer; $\mathrm{DG}=$ dentate gyrus; $\mathrm{Ctx}=$ cortex; $n=6$ per group). (b) Following 1 week of daily paroxetine treatment, a significant upregulation of activin $\beta A$ mRNA could be selectively observed in the hippocampal $\mathrm{DG} ; n=9$ per group). ${ }^{\star} P<0.05$

patients, 329 achieved response after 5 weeks of antidepressant treatment and 246 were non-responders. In all, 166 SNPs within 10 genes belonging to the activin signalling pathway were selected for association with treatment response. rs12082710 genotype, lying within the betaglycan gene (TGFBR3), achieved the best association with a nominal allelic $P$-value of 0.0003 and survived correction for multiple testing with an adjusted $P$-value of 0.044 , odds ratio of 0.65 , confidence interval of $0.51-0.82$ (see Figure 4 for regional association plot of TGFBR3). We re-run PLINK using age, gender and severity of depression at admission as covariate, but observed no significant alteration of the association with response. The homozygote carriers of the major allele were more frequent among responders (responders vs non-responders: $\mathrm{TT}=43.8 \% / 26.8 \%$ ). A repeated-measures ANOVA showed a highly significant interaction between rs12082710 genotype $\left(F_{926,608}=4.8\right.$; $P=9.28 \times 10^{-5}$ ) and time on change in HAM-D score. There was also a significant main effect of rs12082710 genotype $\left(F_{2819,316}=6.7 ; P=0.001\right)$ (see Figure 5). When adding age and gender as a covariate in this repeated-measures analysis, the associations with the SNP remained significant (interaction effect: $F_{926,608}=5.042 ; P=1.97 \times 10^{-5}$ ). Since the severity of depression measured at admission is already a dependent variable of the ANOVA, it was not used as a covariate.
Overall 14 SNPs were nominally associated with treatment response after 5 weeks, 9 of these were located within TGFBR3, but only two of these in strong linkage disequilibrium $\left(r^{2}>0.8\right.$; see Figure 4$)$.

\section{Discussion}

Using a translational approach by combining data from a preclinical mouse model with genetic association data for antidepressant response in patients, we have obtained supportive evidence for a yet undescribed involvement of the activin signalling pathway in modulating antidepressant treatment outcome.

The particular strength of our study lies in the combination of data from mouse experiments mimicking the clinical situation with chronic antidepressant treatment together with genetic association data in a cohort of depressed patients. The combination of animal and human data allowed us to preselect potential candidate genes from the unbiased microarray approach for a more in-depth analysis in an association study for antidepressant treatment response in depressed patients and to increase the power of the association study by limiting the number of tests. ${ }^{24}$

Based on a hypothesis-free strategy, we searched for novel antidepressant target genes and identified activin $\beta \mathrm{A}$, a member of the transforming growth factor $\beta$ superfamily as one of the genes most strongly upregulated by paroxetine in the mouse hippocampus. ${ }^{12}$ During the past years, more and more findings revealing neuroplastic and complex adaptive effects of antidepressant drugs have begun to challenge the 'monoamine hypothesis of depression (for a review see Berton and Nestler ${ }^{25}$ and Millan ${ }^{26}$ ). Activin $\beta A$, a member of the transforming growth factor $\beta$ superfamily, was originally characterized based on its ability to modulate secretion of follicle-stimulating hormone from the pituitary. ${ }^{27,28}$ Later, it has been shown that activin $\beta A$ supports neuron survival in primary cell cultures of rat hippocampus and facilitates neuronal development. ${ }^{29,30}$

The activin pathway may be an antidepressant target with a potential more rapid onset of action. Subchronic as well as chronic treatment with paroxetine resulted in a significant upregulation of activin $\beta A$ mRNA in the CA1 region and the dentate gyrus of the hippocampus. Concomitantly, we observed an upregulation of activin receptor type IA mRNA in the dentate gyrus and the CA3 area.

In contrast, single paroxetine administration did not change activin $\beta A$ mRNA levels in the mouse brain, indicating that the paroxetine-induced time course of activin A induction parallels the clinically well-known delay in the onset of action of antidepressant effects.

Antidepressants with a more rapid onset of action have been the subject of intense research interest for many years, as those would have an enormous impact on the burden of depression and, consequently, on public health. ${ }^{31}$ Our data suggest that compounds directly targeting the activin pathway could prove to be antidepressants with a more rapid onset of action. A very recent study by Malki and co-workers ${ }^{32}$ applied a comparable translational approach for the identification of novel antidepressant-responsive genes; however, these 
a

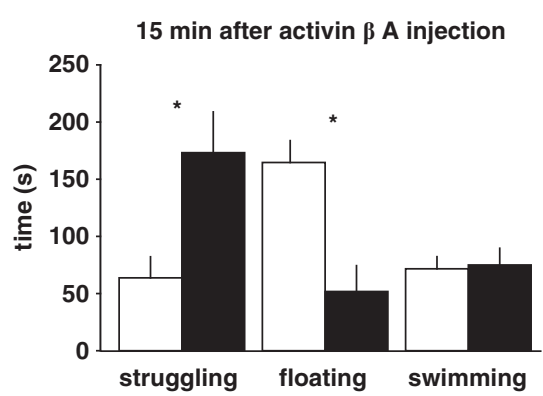

b

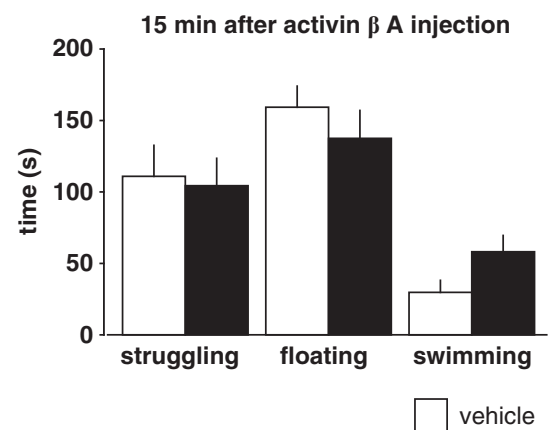

Dentate gyrus

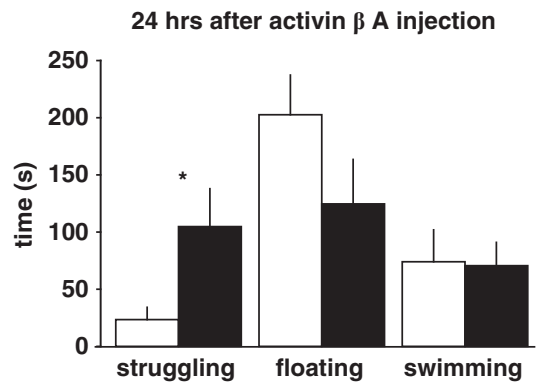

Amygdala

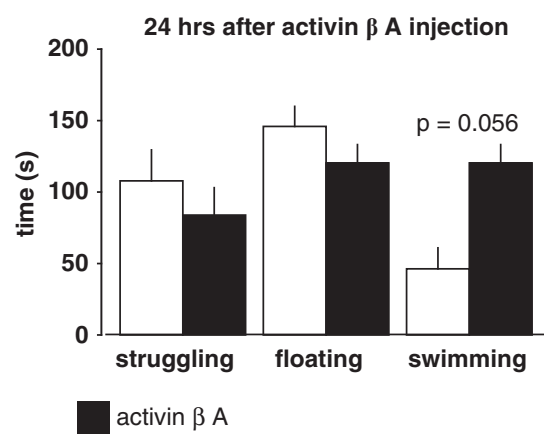

Figure 3 Stereotactic infusion of activin A into the hippocampal dentate gyrus exerts antidepressant-like behavioural effects in the forced swim test (FST) paradigm. (a) A strong antidepressant-like effect of activin A injection into the hippocampal dentate gyrus could be observed: animals bilaterally injected with activin A showed a significant increase in struggling $\left({ }^{*} P<0.05\right)$ and a significant decrease in floating behaviour $\left({ }^{*} P<0.01\right)$ with no change in swimming 15 min after treatment. The significant difference in struggling behaviour between the treatment groups could still be observed $24 \mathrm{~h}$ after activin $\mathrm{A}$ injection. To investigate whether the behavioural effects of activin $\mathrm{A}$ injection are dependent on the neuroanatomical region, we bilaterally injected activin A into the amygdala (b) Acute injection of activin A into the amygdala did not significantly alter any of the behavioural parameters measured in the FST. However, $24 \mathrm{~h}$ after the activin A injection, we observed a trend $(P=0.056)$ towards an increase in swimming behaviour in animals that had been injected with activin $\mathrm{A}$ into the amygdala on the day before.

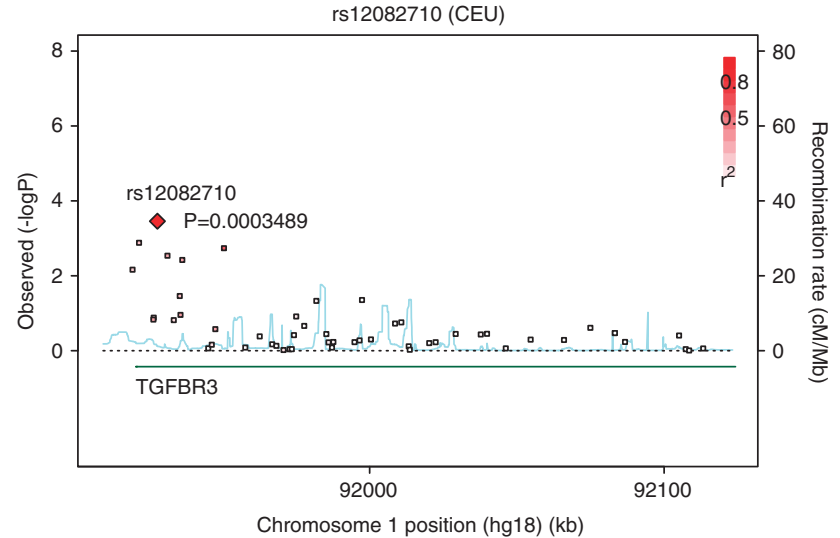

Figure 4 Association of single-nucleotide polymorphisms (SNPs) in TGFBR3 and response to antidepressant treatment. The -log $P$-values, allelewise ( $y$ axis), are plotted against physical location of the SNPs on chromosome 1 ( $x$ axis). Two red markers: markers in strong linkage disequilibrium $\left(\mathrm{LD} r^{2}>0.8\right)$.

authors used a different design for the mouse experiment with antidepressant treatment duration varying between 1 and 14 days.

In addition to its neuroprotective properties, activin was shown to increase the number of synaptic contacts and the length of dendritic spines by modulating actin dynamics. ${ }^{33}$ While the precise nature of the relationship between the pathophysiology of major depression and possible dysfunction of neuroplasticity still remains poorly understood, there is good evidence to suggest that upregulation of neurotrophic factors by antidepressants might contribute to long-term adaptations and synaptic remodelling that are required for the therapeutic actions of these agents. In this context, the strong paroxetine-induced upregulation of activin $A$ in the mouse hippocampus is of particular interest. A potential involvement of activin $\beta A$ in the mechanism of antidepressant action is supported by previous work showing an increase in the rat hippocampus following acute and chronic electroconvulsive seizures (ECS); however, this study failed to identify activin to be upregulated by antidepressant treatment with desipramine or fluoxetine. ${ }^{34}$

Infusion of activin A into the dentate gyrus elicits pronounced antidepressant-like behaviour. In addition to its neurotrophic and neuroprotective properties, there is only very limited data supporting an involvement of activin A in the modulation of depression-related emotional behaviour to date. We, therefore, investigated the behavioural effects of stereotactic injection of activin A into two different brain regions known to have a key role in the modulation of depression-like behaviour, that is, the hippocampus and the amygdala. Both regions have been implicated in the pathophysiology of human affective disorders. ${ }^{35}$ In addition, both regions are known to be richly endowed with activin 


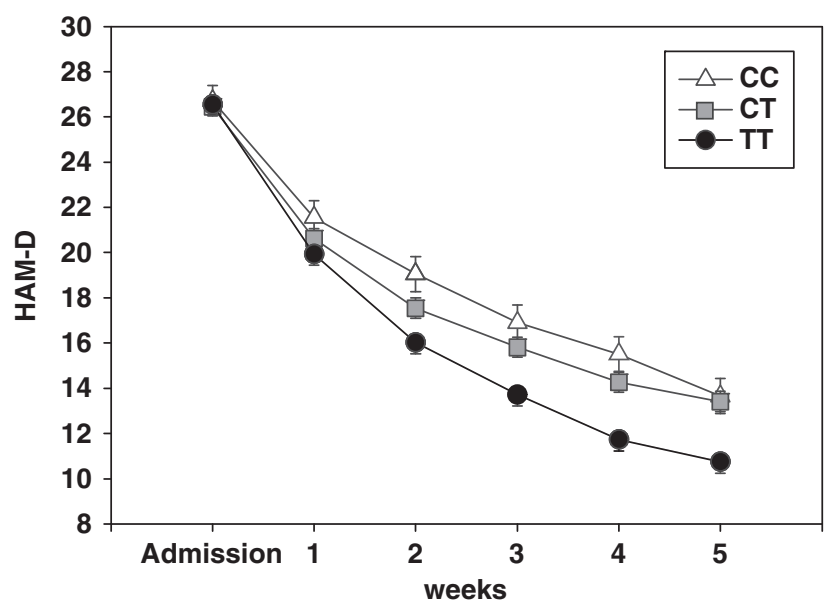

Figure 5 Genetic association study: association of TGFBR3 SNP rs 12082710 genotype with response over 5 weeks treatment with antidepressants after admission to hospital. Hamilton Depression Rating Scale (HAM-D) scores over the first 5 weeks of hospitalization plotted against the rs12082710 genotype $(n=568$; CC $n=90 ;$ CT $n=272 ;$ TT $n=206$ ). A repeated-measures analysis of variance (ANOVA) showed a significant interaction between rs12082710 genotype and time on HAM-D score $\left(P=9.28 \times 10^{-5}\right)$, as well as a significant main effect of rs12082710 genotype $(P=0.001)$.

type I and II receptors. ${ }^{36}$ Stereotactic infusion of activin A into the dentate gyrus elicited potent antidepressant-like effects in the FST: mice injected with activin A showed a significant increase in active coping strategies without any influence on basal locomotor activity. Those antidepressant-like effects of activin A could still be observed $24 \mathrm{~h}$ after injection. With respect to the described potency of activin to regulate synaptic plasticity by modulating actin dynamics, ${ }^{33}$ those lasting antidepressant-like effects of activin are of particular interest. In agreement with our findings, antenatal antidepressant treatment with serotonin reuptake inhibitors has most recently been shown to increase activin A concentrations in maternal blood, in amniotic fluid and in fetal cord blood. $^{37}$

Further, the antidepressant-like properties of activin A are region-dependent: bilateral infusion of activin $A$ into the amygdala did not alter the behavioural outcome in the FST. We therefore hypothesize that the involvement of the activin system in antidepressant mechanisms of action is regionspecific. Although Dow et al. ${ }^{34}$ failed to show an antidepressant-induced upregulation of activin mRNA, their data on the acute behavioural effects of stereotactic infusion of activin $A$ into the rat brain fit with our results. ${ }^{34}$

While the acute antidepressant-like properties of hippocampal activin $A$ injection are remarkably strong, the neurobiological mechanisms underlying the increase in active coping strategies in the FST remain to be elucidated. A study investigating transgenic mice expressing a dominant-negative mutant of activin IB receptor in the forebrain suggested that activin $A$ is a modulator of GABAergic neurotransmission. $^{38}$ If modulation of GABAergic neurotransmission by activin indeed can be identified as the key mechanism underlying its antidepressant-like effects in future studies, the activin system as an alternative pathway to tune
GABAergic neurotransmission might be a promising target to develop improved and innovative treatments for human affective disorders in the future.

Genetic polymorphisms in the activin signalling pathway predict antidepressant response in patients with major depression. To extend the previously identified associations of TGFBR3 with antidepressant response, ${ }^{13}$ we finally investigated the association between genes involved in the activin pathway and antidepressant response in an extended cohort of depressed patients from the MARS study $(N=575)$ and using a denser tagging SNP set than reported previously. We tested the association of 127 tagging SNPs in 10 genes belonging to the activin pathway. In all, 16 SNPs across three genes (Smad3, TGFBR3 and ACVR1B) showed a nominally significant association with antidepressant response, of which one SNP in the TGFBR3 (rs12082710 genotype) gene remained significant after correction for multiple testing, supporting the previously identified associations of this gene with antidepressant response. In addition to association with the dichotomous responder status, this SNP also showed a very significant association with the change in depressive symptoms over time $(P=0.001$; see Figure 5$)$. In addition, a number of other SNPs in only low-to-moderate linkage disequilibrium with rs12082710 genotype showed nominal associations, further supporting an involvement of genetic polymorphisms in this locus with treatment response (see Figure 4).

TGFBR3 encodes betaglycan (also known as type III receptor for transforming growth factor beta), which is a coreceptor that mediates signalling by transforming growth factor beta (TGF $\beta$ ) superfamily members, including the distinct and often opposed actions of TGF $\beta$ s and inhibins. During the past years, distinct inhibin and TGF $\beta$ bindings sites have been identified on betaglycan, ${ }^{39}$ allowing to separate the co-receptor actions of betaglycan toward inhibin and TGF $\beta$. Lewis et al. ${ }^{14}$ could show that betaglycan can function as an inhibin co-receptor with the activin receptor II. ${ }^{14}$ Betaglycan binds inhibin with high affinity and facilitates inhibin antagonism of activin signalling in cells co-expressing ActRII and betaglycan. Thus, one could speculate that genetic variation in the betaglycan gene modulates activin signalling which, in turn, influences the response to antidepressants as observed in our patient population. So far, mutations in the betaglycan gene have been linked to specific forms of human cancer, such as, for example, renal cell carcinoma, and this loss of betaglycan function has been proposed to impair TGF $\beta 2$ signalling. ${ }^{40}$ In contrast, involvement of betaglycan acting as co-receptor in the inhibin/activin pathway has not yet been implicated in human disorders of the central nervous system nor treatment response so far.

In summary, we provide convergent evidence from combined animal and human genetics data that genes in the activin signalling pathway are interesting candidates involved in the neurobiological mechanisms underlying antidepressant mechanisms of action and treatment response. Moreover, our data suggest that compounds specifically targeting the activin pathway could be antidepressants with a more rapid onset of action. Although the cellular and molecular mechanisms mediating treatment response still need to be characterized 
in more detail, we are confident that the here presented, mutually supportive evidence from both preclinical and clinical studies makes this pathway a strong and promising candidate for future experimental approaches.

\section{Conflict of interest}

The authors declare no conflict of interest.

1. Andlin-Sobocki P, Wittchen HU. Cost of affective disorders in Europe. Eur J Neurol 2005; 12: 34-38.

2. Kessler RC, Berglund P, Demler O, Jin R, Koretz D, Merikangas KR et al. The epidemiology of major depressive disorder: results from the National Comorbidity Survey Replication (NCS-R). JAMA 2003; 289: 3095-3105.

3. Nemeroff CB, Owens MJ. Treatment of mood disorders. Nat Neurosci 2002; 5: 1068-1070.

4. Duman R. A silver bullet for the treatment of depression? Neuron 2007; 55: 679-681.

5. Agid Y, Buzsaki G, Diamond DM, Frackowiak R, Giedd J, Girault J-A et al. How can drug discovery for psychiatric disorders be improved? Nat Rev Drug Discov 2007; 6: 189-201.

6. Binder EB, Salyakina D, Lichtner P, Wochnik GM, Ising M, Pütz B et al. Polymorphisms in FKBP5 are associated with increased recurrence of depressive episodes and rapid response to antidepressant treatment. Nat Genet 2004; 36: 1319-1325.

7. Uhr M, Tontsch A, Namendorf C, Ripke S, Lucae S, Ising M et al. Polymorphisms in the drug transporter gene $A B C B 1$ predict antidepressant treatment response in depression. Neuron 2008; 57: 203-209.

8. Serretti A, Artioli $P$, Quartesan R. Pharmacogenetics in the treatment of depression: pharmacodynamic studies. Pharmacogenet Genomics 2005; 15: 61-67.

9. Serretti A, Kato A, De Ronchi D, Kinoshita T. Meta-Analysis of serotonin transporter gene promotor polymorphisms (5-HTTLPR) association with selective serotonin reuptake inhibitor efficacy in depressed patients. Mol Psychiatry 2007; 12: 247-257.

10. Horstmann S, Binder EB. Pharmacogenomics of antidepressant drugs. Pharmacol Ther 2009; 124: 57-73.

11. Kato M, Serretti A. Review and meta-analysis of antidepressant pharmacogenetic findings in major depressive disorder. Mol Psychiatry 2010; 15: 473-500.

12. Sillaber I, Panhuysen M, Henniger MSH, Ohl F, Kühne $C$ et al. Profiling of behavioural changes and hippocampal gene expression in mice chronically treated with the SSRI paroxetine. Psychopharmacology (Berl) 2008; 200: 557-572.

13. Ising M, Lucae S, Binder EB, Bettecken T, Uhr M, Ripke S et al. A genomewide association study points to multiple loci that predict antidepressant drug outcome in depression. Arch Gen Psychiatry 2009; 66: 966-975.

14. Lewis KA, Gray PC, Blount AL, MacConell LA, Wiater E, Bilezikjian LM et al. Betaglycan binds inhibin and can mediate functional antagonism of activin signalling. Nature 2000; 404: 411-414.

15. Schmidt MV, Sterlemann V, Ganea K, Alam S, Harbich D, Greetfeld M et al. Persistent neuroendocrine and behavioural effects of a novel, etiologically relevant mouse paradigm for chronic social stress during adolescence. Psychoneuroendocrinology 2007; 32: 417-429.

16. Schmidt MV, Oitzl MS, Levine S, de Kloet ER. The HPA system during the postnatal development of CD1 mice and the effects of maternal deprivation. Brain Res Dev Brain Res 2002; 139: 39-49.

17. Ohl F, Holsboer F, Landgraf R. The modified hole board as a differential screen for behavior in rodents. Behav Res Methods Instrum Comput 2001; 33: 392-397.

18. Porsolt RD, Le Pichon M, Jalfre M. Depression: a new animal model sensitive to antidepressant treatments. Nature 1977; 266: 730-732.

19. Hennings JM, Owashi T, Binder EB, Horstmann S, Menke A, Kloiber S et al. Clinical characteristics and treatment outcome in a representative sample of depressed inpatients-findings from the Munich Antidepressant Response Signature (MARS) project.. J Psychiatric Res 2009; 43: 215-229.
20. Kohli M, Lucae S, Sämann P, Schmidt MV, Demirkan A, Hek K et al. The neuronal transporter gene SLC6A15 confers risk to depression. Neuron 2011; 70: 252-265.

21. Hamilton M. A rating scale for depression. J Neurol Neurosurg Psychiatry 1960; 23: 56-62.

22. Wigginton JE, Cutler DJ, Abecasis GR. A note on exact tests of Hardy-Weinberg equilibrium. Am J Hum Genet 2005; 76: 887-893.

23. Purcell S, Neale B, Todd-Brown K, Thomas L, Ferreira M, Bender D et al. PLINK: a tool set for whole-genome association and population-based linkage analyses. Am J Hum Genet 2007; 81: 559-575.

24. Malhotra AK. The pharmacogenetics of depression: enter the GWAS. Am J Psychiatry 2010; 167: 493-495

25. Berton O, Nestler EJ. New approaches to antidepressant drug discovery: beyond monoamines. Nat Rev Neurosci 2006; 7: 137-151.

26. Millan MJ. The role of monoamines in the actions of established and 'novel' antidepressant agents: a critical review. Eur J Pharmacol 2004; 500: 371-384.

27. Vale W, Rivier J, Vaughan J, McClintock R, Corrigan A, Woo W et al. Purification and characterization of an FSH releasing protein from porcine ovarian follicular fluid. Nature 1986; 321: 776-779.

28. Ling N, Ying SY, Ueno N, Shimasaki S, Esch F, Hotta M et al. Pituitary FSH is released by a heterodimer of the beta-subunits from the two forms of inhibin. Nature 1986; 321: 779-782.

29. Iwahori Y, Saito H, Torii K, Nishiyama N. Activin exerts a neurotrophic effect on cultured hippocampal neurons. Brain Res 1997; 760: 52-58.

30. Trudeau VL, Theodosis DT, Poulain DA. Activin facilitates neuronal development in the rat amygdala. Neurosci Lett 1997; 237: 33-36.

31. Machado-Vieira R, Salvadore G, Luckenbaugh DA, Manji HK, Zarate CA Jr. Rapid onset of antidepressant action: a new paradigm in the research and treatment of major depression. J Clin Psychiatry 2009; 69: 946-958.

32. Malki K, Uher R, Paya-Cano J, Binder E, Rietschel M, Zobel AW et al. Convergent animal and human evidence suggests a role of PPM1A gene in response to antidepressants. Biol Psychiatry 2011; 69: 360-365.

33. Shoji-Kasai Y, Ageta H, Hasegawa Y, Tsuchida K, Sugino H, Inokuchi K. Activin increases the number of synaptic contacts and the length of dendritic spine necks by modulating spinal actin dynamics. J Cell Sci 2007; 120: 3830-3837.

34. Dow AL, Russell DS, Duman RS. Regulation of activin mRNA and Smad2 phosphorylation by antidepressant treatment in the rat brain: effects in behavioral models. J Neurosci 2005; 25: $4908-4916$.

35. Campbell S, Macqueen G. The role of the hippocampus in the pathophysiology of major depression. J Psychiatry Neurosci 2004; 29: 417-426.

36. Bengtsson H, Soderstrom S, Ebendal T. Expression of activin receptors type I and II only partially overlaps in the nervous system. NeuroReport 1995; 7: 113-116.

37. Bellissima V, Visser GH, Verwers TF, van Bel F, Termote JU, van der Heide $M$ et al. Antenatal maternal antidepressant drugs affect Activin A concentrations in maternal blood, in amniotic fluid and in fetal cord blood. J Matern Fetal Neonatal Med 2011; 24: 31-34.

38. Zheng F, Adelsberger H, Muller MR, Fritschy JM, Werner S, Alzheimer C. Activin tunes GABAergic neurotransmission and modulates anxiety-like behavior. Mol Psychiatry 2008; 14: $332-346$

39. Wiater E, Harrison CA, Lewis CA, Gray PC, Vale WW. Identification of distinct inhibin and transforming growth factor beta-binding sites on betaglycan. J Biol Chem 2006; 281: 17011-17022.

40. Copland JA, Luxon BA, Ajani L, Maity T, Campagnaro E, Guo H et al. Genomic profiling identifies alterations in TGFbeta signalling through loss of TGF beta receptor expression in human renal cell carcinogenesis and progression. Oncogene 2003; 22: 8053-8062.

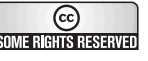

Translational Psychiatry is an open-access journal published by Nature Publishing Group. This work is licensed under the Creative Commons Attribution-NonCommercial-No Derivative Works 3.0 Unported License. To view a copy of this license, visit http://creativecommons.org/licenses/by-nc-nd/3.0/

Supplementary Information accompanies the paper on the Translational Psychiatry website (http://www.nature.com/tp) 